\title{
The Influence of Cholinergic System on Rheumatoid Arthritis
}

\section{Mehranfard D* and Hamurtekin E}

Faculty of Pharmacy, Eastern Mediterranean University, Cyprus

*Corresponding author: Danial Mehranfard, Pharm.D., Faculty of Pharmacy, Eastern, Mediterranean University, Famagusta-Cyprus, Tel: +1(786)409-9084; Email: dm.daniel73@gmail.com

\section{Review Article \\ Volume 3 Issue 1}

Received Date: January 10, 2019

Published Date: January 18, 2019

DOI: $10.23880 /$ oajpr-16000170

\section{Abstract}

Rheumatoid arthritis is one of the most prevalent immune-mediated arthritis and is categorized as a chronic, inflammatory and autoimmune disease, which mainly affects the cartilage, synovial joint, and bone, the causes of which remain unknown. There are different factors playing a role in RA pathophysiology on the molecular level, like Citrullination, Regulatory T cells, Monocytes, Macrophages, Platelet microparticles, and RASFs, which mainly induce inflammation by producing pro-inflammatory cytokines such as IL-8, IL-6, IL-1, and TNF. We have two types of cholinergic systems (CS) in our body, Neuronal CS, in which Acetylcholine (ACh) act as a neurotransmitter and NonNeuronal CS in which ACh act as a local signalling molecule for cellular proliferation and regulation of cellular function including the cells involved in RA inflammation. In light of the recent studies, the links between the CS and cells involved in RA molecular pathophysiology suggest new approaches for controlling RA, which will be discussed further. First, is the stimulation of the vagus nerve, which plays an essential role in the cholinergic anti-inflammatory pathway through its main neurotransmitter ACh resulting in downregulation of TNF $\alpha$ and inhibition of inflammation. The second strategy is stimulation of CAP by selective activation of $\alpha 7 \mathrm{nAChR}$, which is represented by different types of immune cells, such as T and B-lymphocytes, monocytes, dendritic cells, especially macrophages, and FLS through acetylcholine or its selective agonist resulting in decreased TNF production and reduced inflammation. As we discussed previously ACh is one of the necessary components for cellular proliferation especially in RASFs. Choline transporter-like proteins 1 and 2 are accountable for bringing extracellular choline inside the RASFs, which further will convert to ACh within the cell. Therefore, as the final strategy, we can induce apoptotic cell death in RASFs through preventing choline uptake by CTL1 and CTL2. Blocking of choline uptake through Choline transporter-like proteins 1 and 2, presents a novel target for RA treatment, but further in vivo studies are necessary to examine the role of these transporters as drug targets in RA.

Keywords: Rheumatoid arthritis; Cholinergic system; Vagus nerve; $\alpha 7$ Nicotinic ACh receptor; Choline transporter-like proteins

Abbrevations: ANS: Autonomic Nervous System; VNS: Vagus nerve stimulation; RASFs: Rheumatoid Arthritis
Synovial Fibroblasts; FLSs: Fibroblast-Like Synoviocytes; MMPs: Matrix- Metalloproteinases; HRE: Hypoxia 


\section{Open Access Journal of Pharmaceutical Research}

Response Elements; ACPAs: Anticitrullinated Protein Antibodies; PNS: Parasympathetic Nervous System; SNS: Sympathetic Nervous System; CHT: Choline Transporters; ChAT: Choline Acetyltransferase; nAChR and mAChR: Nicotinic Acetylcholine Receptors or Muscarinic Acetylcholine Receptors.

\section{Rheumatoid Arhritis}

\section{RA Background}

Rheumatoid arthritis or RA is one of the most prevalent immune mediated arthritis and is categorized as a chronic, inflammatory and autoimmune disease, which mainly affects the cartilage, synovial joint, and bone, the causes of which remain unknown [1,2]. Currently data on RA involvement among the general population of the United States is estimated to be $0.3 \%$ to $1.5 \%$, with women being more liable to RA disorder than men [3].

During its severe stages this disease limits the joints motion and function, and continuing synovial inflammation causes gradual destruction of the bone and finally joint deformities [4].

We usually presume that inflammation is the main cause of pain in RA, but it may not be the only factor; Especially in chronic RA pain, CNS may play an important role in its generation and maintenance [5] (Figure 1).

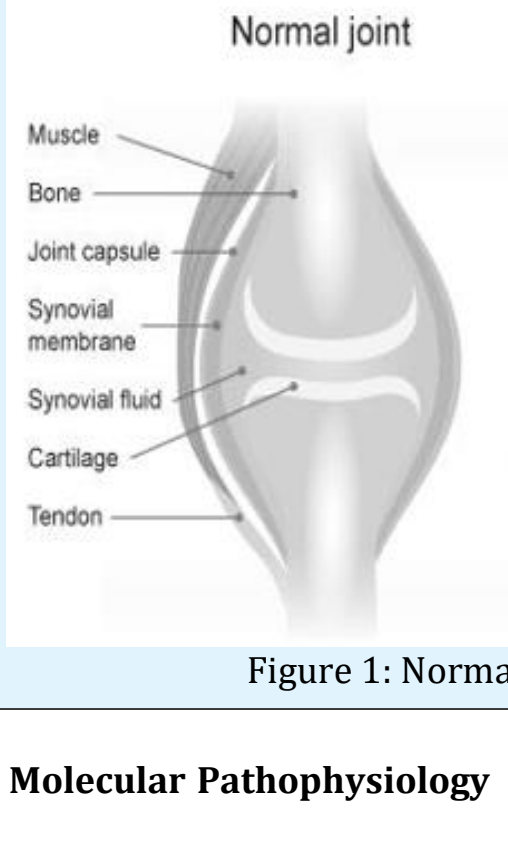

\section{Recent
of RA}

The RA pathophysiology and immune system: In rheumatoid arthritis, memory and naïve B cells penetrate and accumulate in the synovial tissue, in which there seems to be continuous activation of chosen B cell clones, which have great migratory ability $[1,6]$.

Macrophages are playing an important role in RA pathophysiology by releasing pro-inflammatory cytokines including IL-1, IL-6 and TNFa. Chemokine receptor CCR9 expression in RA is vital for the migration to and retention of leukocytes in. Also, it was found that the RA joints had augmented CD14+ number of synovial macrophages and peripheral monocytes $[1,7]$.

Platelet microparticles have been recognized in synovial fluid of RA, where they can stick to surface of leukocytes [1]. Microparticles showed to have membrane bound IL-1a and IL-1b, which triggered FLSs to secrete pro-inflammatory cytokines such as IL-8 and IL-6 [8].

RA synovial fibroblast: Rheumatoid arthritis synovial fibroblasts (RASFs) which are also called Fibroblast-like synoviocytes (FLSs), in hyperplastic rheumatoid synovium are main cell types, in which they have an essential duty in inflammatory cascade augmentation, connecting adaptive and innate immunity [1].

Fibroblast like synoviocytes intimal linings are key factor cells in producing cytokines that maintain inflammation and increase the production of matrixdegrading enzymes like metalloproteinases (MMPs) that contribute to cartilage destruction [9-11]. FLS also release IL-32, which increase TNFa, IL-1 or IL-18 production by macrophages and/or dendritic cells [12]. 


\section{Open Access Journal of Pharmaceutical Research}

In RA, enlarged FLS mass, exceeds oxygen supply, causing build-up of lactate for instance, an anaerobic glycolysis product. Cell response to the hypoxia, by changing and modifying gene expression through hypoxia response elements (HRE) which has effect on the immune and inflammatory pathways, cellular signaling and angiogenesis all of which may give rise to chronic inflammation [13] (Figure 2).

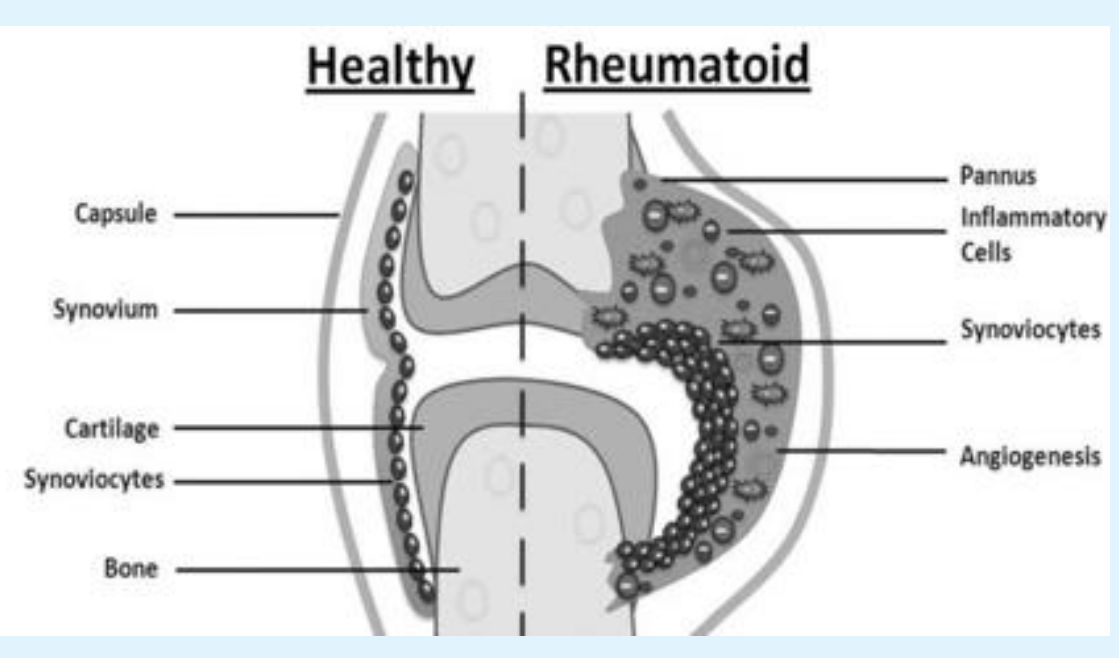

Figure 2: On the left side a healthy joint includes a small amount of synovium and a lining layer of Synoviocytes. On the right sight, in the Rheumatoid joint, immune cells quickly change their place to sublining and by release of chemokines or cytokines they attract more immune cells into the area, causing an inflammatory cycle [14].

Citrullination: Citrullination is a term referring to the modification of the arginine amino acid into citrulline after transcription. In recent years, evidence has suggested a link between RA and anticitrullinated protein antibodies or ACPAs, which currently measure as anticyclic citrullinated peptide autoantibodies. Recently, Anticitrullinated protein antibodies (ACPAs) isolated from RA sera, through binding to $78 \mathrm{kDa}$ glucoseregulated protein or GRP78, a citrullinated surface expressed protein, were shown to increase the activity of $\mathrm{TNFa}$ and NF-kB production in macrophages or monocytes [1].

\section{Cholinergic System}

\section{CS Background}

Acetylcholine, in the brain plays a key role as neurotransmitter, which brings about neuronal transmission in both parasympathetic and sympathetic nervous system (PNS and SNS). Additionally, in the postganglionic parasympathetic/vagal efferent neurons, $\mathrm{ACh}$ is the main neurotransmitter. It acts through
Nicotinic (metabotropic) and Muscarinic (ionotropic) receptors [15].

Studies of NS provide us most recent knowledge about the process of production, storage, metabolism and actions of Acetylcholine. As we know, Acetylcholine is produced from choline brought into the cell through high affinity choline transporters (CHT) and Acetyl-CoA, mainly by choline acetyltransferase or ChAT and slightly by carnitine acetyltransferase. Synthesized Ach is then carried into the synaptic vesicles through vesicular ACh transporter (VAChT) and is stored there until an increase in the free $\mathrm{Ca} 2+$ concentration, which causes exocytosis, and releasing of ACh. After ACh is released, depending on the target, it can act on either nicotinic acetylcholine receptors or muscarinic acetylcholine receptors (nAChR and $\mathrm{mAChR}$ ) [16].

We can find ACh and its synthesizing enzyme, ChAT, within different human cells such as endothelial, epithelial and mesothelial cells, but most importantly in human immune cells such as mast cells and macrophages [17] (Figure 3). 


\section{Open Access Journal of Pharmaceutical Research}

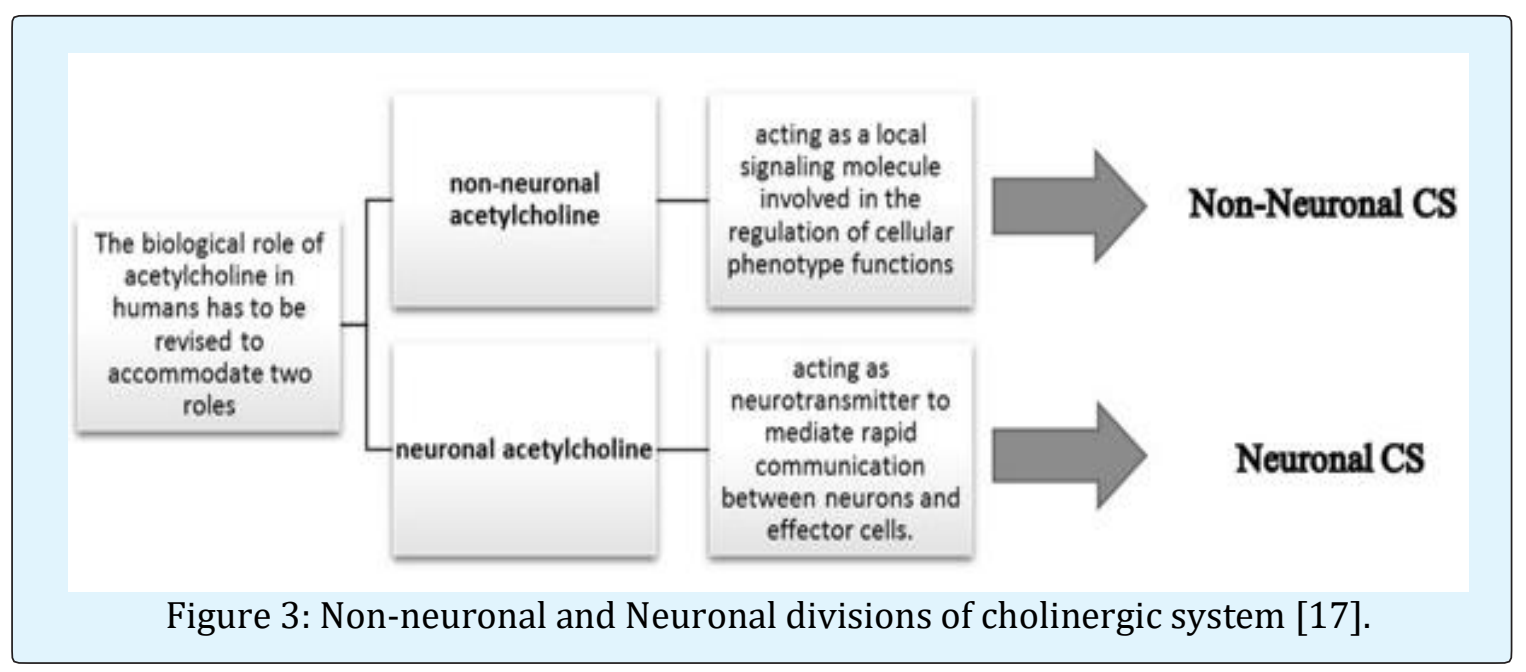

\section{Neuronal Cholinergic System}

First the ANS (Autonomic Nervous System) was introduced; then afterwards SNS namely sympathetic nervous system and PNS or parasympathetic nervous system were introduced respectively [18].

One of the main elements of the parasympathetic nervous system which mostly shows its activity during the resting state of the body is Vagus nerve $[4,18]$.

In both sympathetic and parasympathetic, nervous system pre-ganglionic neurons are cholinergic so synaptic transmission is the same. In synaptic vesicles, ACh is stored and released upon the nerve stimulation (depolarization) by a Ca2+ dependent process. Then Ach stimulates or depolarizes the post synaptic membrane by binding to postsynaptic receptors [18].

At the postganglionic effector sites of the PNS, ACh is the key neurotransmitter, which its postsynaptic receptors are either nicotinic or muscarinic. The majority of its physiologic effects are mediated by Muscarinic receptors. Extracellular enzyme acetylcholinesterase (AChE), rapidly neutralize ACh through hydrolysis upon its release into the synaptic cleft [18].

Typical neurotransmitters of SNS are epinephrine, norepinephrine (NE) and dopamine (DA) which are released by postganglionic neurons and to affect sympathetic physiological responses, they interact with adrenergic receptors [18].

\section{Non-Neuronal Cholinergic System}

ACh acts as a local cell-signalling molecule in nonneuronal cholinergic system [17]. For example, independent of neuronal innervation both immune cells (such as macrophages) and epithelial cells show all elements of cholinergic system. Repeatedly it has been showed that acetylcholine has a role in the adjustment of nervous-independent, fundamental cell functions such as local release of mediators like proinflammatory cytokines and Nitroxide, cellular proliferation and differentiation [17].

On one hand, in neuronal CS, ACh synthesis happens within the nerve terminals, it accumulates and stores in cholinergic vesicles and releases up on the depolarization or stimulation of neuronal cells in high amounts through exocytosis to permit fast neurotransmission and quickly hydrolyze in synaptic terminals.

On the other hand, in non-neuronal CS, ACh synthesis happens approximately across the whole cell, it accumulates in cytosol and is continuously released by transporter mediators, which cause ACh to eliminate slower, and as a result show longer action [17].

\section{The Influence of CS on RA}

\section{Vagus Nerve}

Through Vagus nerve inflammatory reflex, the nervous system plays a significant role in limiting inflammatory responses [19].

As observed in RA patients, the afferent vagus nerve can detect inflammation in peripheral tissues then send this information to the brain and through vagus nerve, brain can show its anti-inflammatory effect in inflamed tissues [4]. This approach is known as Cholinergic Antiinflammatory Pathway or CAP [19]; We can also define CAP as the detection of peripheral inflammation by the 


\section{Open Access Journal of Pharmaceutical Research}

afferent vagus nerve and efferent vagus nerve antiinflammatory answer through the release of its principal neurotransmitter Ach $[4,20]$. This pathway provides a quick and strong mechanism for the host to fight immoderate inflammation in a localized manner [19].

Cutting of the vagus nerve through vagotomy (is a surgical procedure that involves removing part of the vagus nerve) increased inflammation in different animal models: it increased production of systemic tumor necrosis factor $\alpha$ and fastened shock development in the endotoxemia model in rats, also it increased ceruleaninduced acute pancreatitis and inflammation accompanied with bacterial peritonitis locally and systemically in mice [19].
A recent study suggested a potential role for Vagus nerve stimulation (VNS) in the treatment of RA through showing anti-inflammatory effect of Vagus nerve stimulation on carrageen-induced paw inflammation $[20,21]$. Also new experiments have shown the ability of VNS in Collagen-induced arthritis (CIA) development suppression in rats $[20,22]$.

In the rat model, Vagotomy without stimulation of Vagus nerve followed by IV endotoxin administration, significantly increased the level of TNF in liver and serum, which suggests to us a direct role for efferent Vagus neurons in the regulation of TNF production [23] (Figure 4).

\section{Electrical Stimulation of Vagus Nerve}

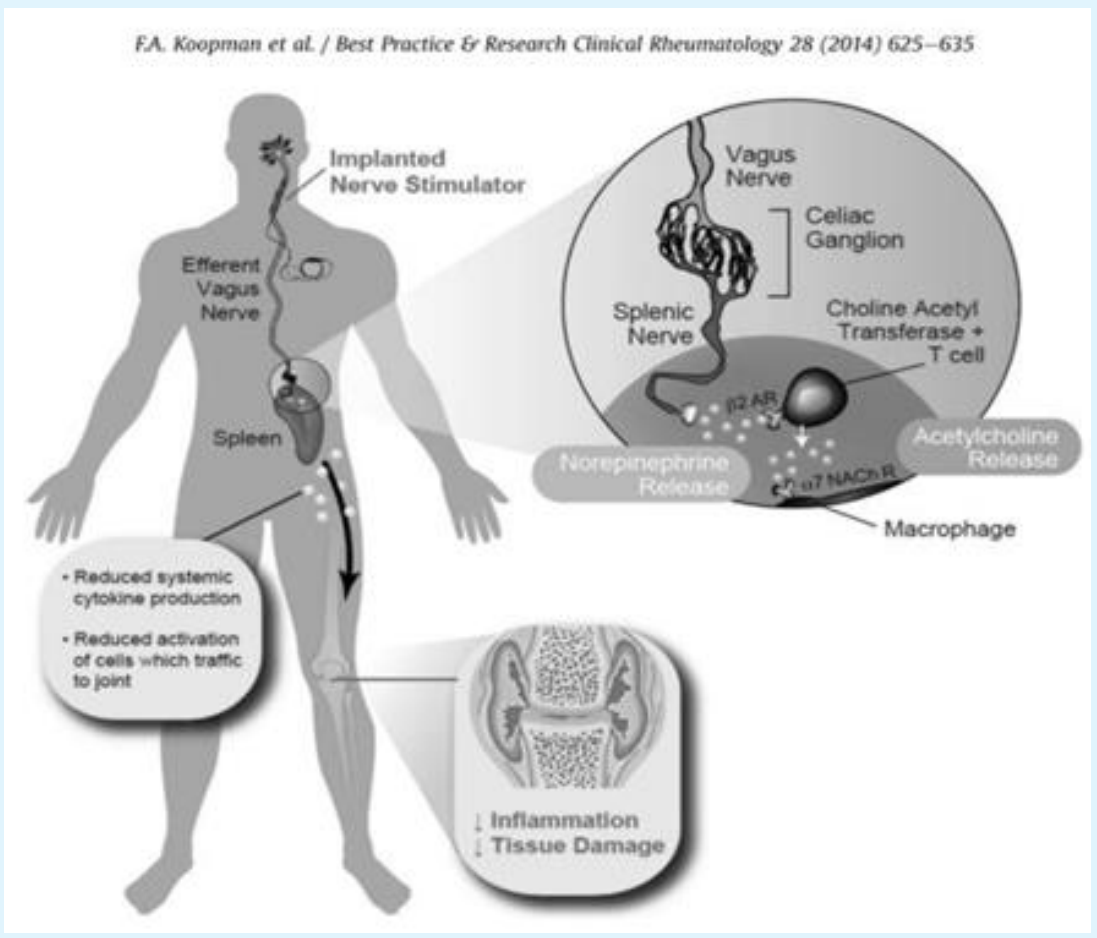

Figure 4: Parasympathetic Vagus nerve electrical stimulation cause activation of the sympathetic splenic nerve via secretion of norepinephrine, which cause choline acetyltransferase positive T-cells to produce acetylcholine and reduce joint inflammation by decrease cytokine production through binding to the nicotinergic acetylcholine receptor type 7 (a7nAChR) [4].

It has recently been shown that during inflammation vagus nerve has an essential role in suppressing cytokine expression. Either by pharmacological or electrical methods, stimulating the vagus nerve increases survival rate in animal model of inflammation [24].
Europe in 1994 and 3 years after US approved Vagus Nerve Stimulation therapy or VNS as a treatment for epilepsy; it was also approved for depression treatment in the US. Following neurosurgical implantation of a vagus nerve stimulator, VNS can be performed [4]. 


\section{Open Access Journal of Pharmaceutical Research}

Vagus nerve electrical stimulation decreased inflammation through down regulation of TNF $\alpha$ production and preserved endotoxemia rats from hypotension [25]. VNS did not allow the severe inflammatory response to hypovolemic hemorrhagic shock, [26] and reduced intestinal inflammation in mouse model of postoperative ileus [27]. So, we can conclude that VNS prevented systematic inflammation and decreased lethality [20].

Spleen role in anti-inflammatory effect of VN: Antiinflammatory mediator of the CAP, Acetylcholine, which is also a PNS neurotransmitter, shows its anti-inflammatory activity through activation of a7nAChR. Vagus nerve cells and also non-neuronal cells such as the spleen are able to produce ACh [4].

In the splenectomized mice (Splenectomy is a surgery to remove the entire spleen) treated with endotoxin, VNS was not able to decrease the serum high TNF level, which suggests a role for the spleen in the vagus nerve antiinflammatory activity [28].

Recently, it has been shown that stimulation or inhibition of $\alpha 7 \mathrm{nAChR}$, particularly affects TNF $\alpha$ production by the spleen [28-30]. Since it has been hypothesized that CAP function is largely depends on the spleen [30], TNF $\alpha$ production by splenocytes investigated in $\alpha 7 \mathrm{nAChR}-/-$ mice and wild-type (WT) litter-mates [31]. It was an increase in TNF $\alpha$ production of splenocytes of $\alpha 7 \mathrm{nAChR}$ /- mice. As TNF $\alpha$ has an essential role in RA pathogenesis this may partially explain the observed exacerbation of Arthritis. [32,33].

Following VNS, through the sympathetic splenic nerve, the anti- inflammatory response seems to move towards the spleen. The splenic nerve secretes NE, which stimulates choline acetyltransferase-positive $\mathrm{T}$ cells (CHAT+ T cells) in the spleen to produce ACh. Within the RA synovium CHAT+ cells are also found, which implies that in the joint, ACh can also be produced locally [4].

According to above studies we can conclude that the parasympathetic nervous system by adjusting immune cells in the spleen might regulate systemic inflammation [20].

\section{Nicotinic Acetylcholine Receptor ( $\alpha 7 \mathrm{nAChR})$}

$\mathrm{ACh}$, the main neurotransmitter of the vagus nerve, is key anti-inflammatory mediator of the CAP. It especially interacts with $\alpha 7$ sub-unit of the nicotinic acetylcholine

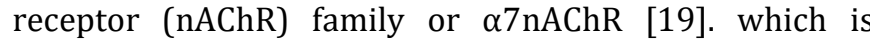
expressed by some of the cells participated in inflammatory response including macrophages [20,25]. $\mathrm{ACh}$, concentration dependently reduces TNF production by human macrophages after binding to the $\alpha 7 \mathrm{nAChR}$ [23].

In animal models of pancreatitis, selective nAChR agonists have showed effectiveness reducing inflammation through decreasing production of cytokines by macrophage. [19] On the other hand, in the mice lacking the $\alpha 7$ subunit, isolated peritoneal macrophages do not answer to Ach and nicotine, and in the presence of this cholinergic agonists still continue to produce TNF [23].

Through the relatively high permeability of $\alpha 7$ subunit to $\mathrm{Ca} 2+$ from the other nAChRs, its extensive expression in the CNS and PNS and its sensitivity and quick desensitization to antagonists like $\alpha$-bungarotoxin, identification of $\alpha 7$ subtype is possible [22].

$\alpha 7 \mathrm{nAChR}$ mechanism of action: Activation of $\alpha 7 \mathrm{nACh} R$ has an anti-inflammatory effect in human macrophages through:

A. JAK2-STAT3 signaling pathway. Modified JAK-STAT signaling seems to have a significant role in RA as inflamed joints have showed increased expression of STATs [20].

B. Prevention of activation of the NFKB pathway [20].

a7nAChR agonists: Non-specificity and toxicity of Nicotine limited its usage, so we can use $\alpha 7 n A C h R$ agonists as more effective options for RA treatment through CAP stimulation by selective activation of this receptor [20] (Table 1).

\begin{tabular}{|c|c|}
\hline $\boldsymbol{\alpha} 7$ Agonists & Activity \\
\hline AR-R17779 & $\begin{array}{c}\text { Selective and potent } \alpha 7 \mathrm{nAChR} \\
\text { full agonist }\end{array}$ \\
\hline PNU-282987 & $\begin{array}{c}\text { Selective and potent } \alpha 7 \mathrm{nAChR} \\
\text { full agonist }\end{array}$ \\
\hline GTS-21 & $\alpha 7 \mathrm{nAChR}$ partial agonist \\
\hline
\end{tabular}

Table 1: Selective $\alpha 7 \mathrm{nAChR}$ agonists [20].

\section{AR-R17779}

AR-R17779 has been tasted in Collagen-induced arthritis or CIA and was shown to reduce inflammation [31], because of its poor blood-brain barrier penetration it suggests that AR-R17779 anti-inflammatory activity most probably is because of peripheral stimulation of $\alpha 7$ nAChR [20]. 


\section{Open Access Journal of Pharmaceutical Research}

Following AR-R17779, Ach or Nicotine pre-treatment, FLS of RA patients through activation of $\alpha 7 \mathrm{nAChR}$ decrease IL-8 and IL-6 production [20,34-36] (Figure 5).

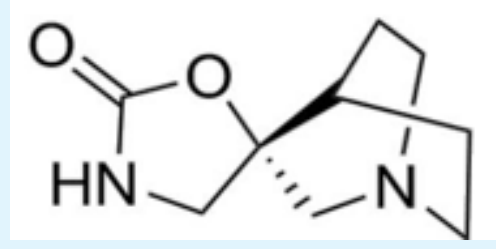

Figure 5: Chemical structure of AR-R17779.

\section{PNU-282987}

PNU-282987 has been shown to dose-dependently inhibit IL-6 production by FLS, as well as reduced IL-6 production in IL-1 stimulated FLS [35,37], it also mimicked acetylcholine anti-inflammatory effect on FLS [35] (Figure 6).

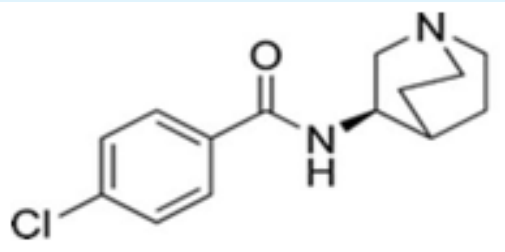

Figure 6: Chemical structure of PNU-282987.

\section{GTS-21}

GTS-21 is the only $\alpha 7 \mathrm{nAChR}$ agonist, which has been tested in humans. It was orally administered to Alzheimer disease patients, schizophrenia patients and healthy volunteers. According to obtained data, doses of up to $450 \mathrm{mg}$ per day were finely tolerated with no significant adverse effects [20,38] (Figure 7).

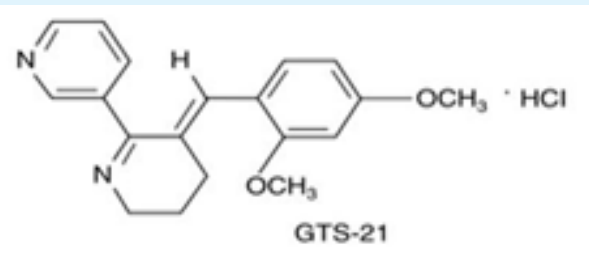

Figure 7: Chemical structure of GTS-21.

\section{CTL1- \& CTL2-Mediated Choline Transport System}

In the majority of the non-neuronal CS cells, Acetylcholine is directly released by transporters instead of being stored in vesicles [39]. We have three families of choline transporter system: transporter 1 (CHT1), polyspecific organic cation transporters (OCT1-2) which show low affinity for choline, and choline transporter-like proteins (CTL1-5) [9,40,41].

All essential ingredients for transporting choline into the cell and releasing of Ach are present in the OCT family and all of CTL family members and were expressed in cartilage and synovial. CTL1 and CTL2 expression, was limited to fibroblasts and synovial macrophages [39]. While all CTL family members have been shown to be expressed in human joint synovial tissue and cartilage, CTL1 is the most obviously expressed in both cartilage and synovia [39]. Also, according to recently conducted experiments [9], in RASFs, mRNAs of CTL1 and CTL2 were greatly expressed, while mRNAs of CTL3 and OCT3 were negligibly expressed [9].

In CTL1 and CTL2 expressed cells, the existence of carnitine acetyltransferase (CarAT) which is an acetylcholine-synthesizing enzyme provides evidence that shows production of ACh by non-neuronal synovial cells. So, all required elements for ACh synthesize and release are existing in the human joint synovial tissue and cartilage [39].

Properties of [3H] choline uptake in RASFs: Uptake of [3H] Choline into RASFs is moderated through high- and low- affinity transporter systems [9].

CTL1, PH dependently uptake choline [40-43]. [3H] Choline uptake in RASFs under different extracellular $\mathrm{pH}$ was examined, as a result [3H] choline uptake was increased under higher extracellular medium $\mathrm{PH}$ and decreased under lower PH. CTL2 is practically expressed in RASFs similar to CTL1 and in these cells is accountable for choline uptake. Therefore, we can say, CTL1 and CTL2, respectively, are high- and low- affinity choline transporters in RASFs. CTL2 or low-affinity choline transporter assumed to have a key role in choline transport into RASFs, which need large amount of choline for cell proliferation; also, it appears to help with choline uptake during the CTL1 dysfunction [9].

Choline transporter-like proteins, new target in RA treatment: Expression of CTL1 and CTL2 in cartilage and synovial tissue of RA patients was restricted to fibroblasts and synovial macrophages and seems to be associated with uptake of choline in those cells. In the human joints, CTL1 and CTL2 expression can show that these transporters play a role in non-neuronal ACh synthesis and release [39]. As we discussed we can conclude that under the normal physiological conditions, high-affinity CTL1 and under pathophysiological conditions, lowaffinity CTL2 may be involved in choline transport [9]. 


\section{Open Access Journal of Pharmaceutical Research}

Choline is one of the necessary components for cellular proliferation; therefore, choline deficiency can decrease the proliferation of these cells and increase apoptosis. The rate-limiting step in choline phospholipid metabolism is intracellular choline uptake by both CTL1 and CTL2 in RASFs and a requirement for RASF proliferation, so, by obstructing choline uptake through CTL1 and CTL2 we can induce apoptotic cell death in RASF [9].

\section{Conclusion}

A fuller understanding of the links between the CS and cells involved in RA pathophysiology suggests new practical pathways for either controlling or treating RA but they require further in vivo studies. In non-neuronal $\mathrm{CS}, \mathrm{ACh}$ acts as a local signaling molecule involved in the adjustment of the cellular function and more importantly cellular proliferation including the cells involved in RA inflammation; by vagus nerve stimulation, we can activate CAP through its main neurotransmitter ACh, which results in reducing of pro-inflammatory cytokines production such as TNF $\alpha$ and inhibiting inflammation. Stimulation of CAP through selective activation of $\alpha 7 \mathrm{nAChR}$, which is expressed by various immune cells, including macrophages and FLS through ACh or its selective agonists, results in down regulation of proinflammatory cytokines production and reducing of inflammation. There are three pharmacological $\alpha 7 \mathrm{nAChR}$ agonist available two of which (AR-R17779 and PNU282987) are acting as full agonism and one of them (GTS21) as partial agonism. However, because of the lack of testing on humans (except GTS-21) there is need for further studies. Choline is one of the necessary components for cellular proliferation, in light of recent studies we know that in cartilage and synovial tissue of RA patients, expression of CTL1 and CTL2 is limited to fibroblasts and synovial macrophages and seems to be involved in choline uptake in those cells, so, by blocking choline uptake through CTL1 and CTL2 we can cause choline deficiency and decrease the proliferation of these cells and increase apoptosis as result, but further in vivo studies are required to examine the role of these transporters as drug targets in RA.

\section{References}

1. Cooles FAH, Isaacs JD (2011) Pathophysiology of rheumatoid arthritis. Curr Opin Rheumatol 23(3): 233-240.

2. Smolen J, Keystone EC (2012) Rheumatoid arthritis: Where are we now? Pathogenesis, treatment response and tailored therapy. Rheumatol 51(5): 2122.
3. Puttlitz CM, Goel VK, Clark CR, Traynelis VC, Scifert JL, et al. (2000) Biomechanical rationale for the pathology of rheumatoid arthritis in the craniovertebral junction. Spine (Phila $\mathrm{Pa}$ 1976) 25(13): 1607-1616.

4. Koopman FA, Schuurman PR, Vervoordeldonk MJ, Tak PP (2014) Vagus nerve stimulation: A new bioelectronics approach to treat rheumatoid arthritis?. Best Pract Res Clin Rheumatol 28(4): 625635.

5. Lee YC, Nassikas NJ, Clauw DJ (2011) The role of the central nervous system in the generation and maintenance of chronic pain in rheumatoid arthritis, osteoarthritis and fibromyalgia. Arthritis Res Ther 13(2): 211.

6. Scheel T, Gursche A, Zacher J, Häupl T, Berek C (2011) V-region gene analysis of locally defined synovial B and plasma cells reveals selected B cell expansion and accumulation of plasma cell clones in rheumatoid arthritis. Arthritis Rheum 63(1): 63-72.

7. Schmutz C, Cartwright A, Williams H, Haworth O, Williams JH, et al. (2010) Monocytes/ macrophages express chemokine receptor CCR9 in rheumatoid arthritis and CCL25 stimulates their differentiation. Arthritis Res Ther 12(4): R161.

8. Boilard E, Nigrovic PA, Larabee K, Watts GF, Coblyn JS, et al. (2010) Platelets Amplify Inflammation in Arthritis via Collagen-Dependent Microparticle Production. Science 327(5965): 580-583.

9. Seki M, Kawai Y, Ishii C, Yamanaka T, Odawara M, et al. (2017) Functional analysis of choline transporters in rheumatoid arthritis synovial fibroblasts. Mod Rheumatol 27(6): 995-1003.

10. Smolen JS, Aletaha D, Koeller M, Weisman MH, Emery $P$ (2007) New therapies for treatment of rheumatoid arthritis. Lancet 370(9602): 1861-1874.

11. Bartok B, Firestein G (2010) Fibroblast-like synoviocytes: key effector cells in rheumatoid arthritis. Immunol Rev 233(1): 233-255.

12. Alsaleh G, Sparsa L, Chatelus E, Ehlinger M, Gottenberg JE, et al. (2010) Innate immunity triggers IL-32 expression by fibroblast-like synoviocytes in rheumatoid arthritis. Arthritis Res Ther 12(4): R135.

13. Del Rey MJ, Izquierdo E, Usategui A, Gonzalo E, Blanco FJ, et al. (2010) The transcriptional response of 


\section{Open Access Journal of Pharmaceutical Research}

normal and rheumatoid arthritis synovial fibroblasts to hypoxia. Arthritis Rheum 62(12): 3584-3594.

14. Hawtree S, Muthana M, Wilson AG (2013) The role of histone deacetylases in rheumatoid arthritis fibroblast-like synoviocytes. Biochem Soc Trans 41(3): 783-788.

15. Hu SX, Sui HX, Jin HJ, Ni XY, Liu XX, et al. (2012) Lipopolysaccharide and dose of nicotine determine the effects of nicotine on murine bone marrowderived dendritic cells. Mol Med Rep 5(4): 10051010.

16. Kawashima K Fujii $\mathrm{T}$ (2003) The lymphocytic cholinergic system and its contribution to the regulation of immune activity. Life Sci 74(6): 675696.

17. Wessler I, Kilbinger $\mathrm{H}$, Bittinger $\mathrm{F}$, Unger $\mathrm{R}$, Kirkpatrick CJ (2003) The non-neuronal cholinergic system in humans: Expression, function and pathophysiology. Life Sci 72(18-19): 2055-2061.

18. Johnson J0 (2013) Pharmacology and Physiology for Anesthesia, Elsevier Inc., 12. Autonomic Nervous System Physiology pp: 208-217.

19. van Maanen MA, Stoof SP, LaRosa GJ, Vervoordeldonk MJ, Tak PP (2010) Role of the cholinergic nervous system in rheumatoid arthritis: aggravation of arthritis in nicotinic acetylcholine receptor $\alpha 7$ subunit gene knockout mice. Ann Rheum Dis 69(9): 1717-1723.

20. van Maanen MA, Vervoordeldonk MJ, Tak PP (2009) The cholinergic anti-inflammatory pathway: towards innovative treatment of rheumatoid arthritis. Nat Rev Rheumatol 5(4): 229-232.

21. Borovikova LV, Ivanova S, Nardi D, Zhang M, Yang H, et al. (2000) Role of vagus nerve signaling in CNI1493-mediated suppression of acute inflammation. Auton Neurosci Basic Clin 85(1-3): 141-147.

22. Tracey KJ (2002) The Inflammatory Reflex. Nature 420(6917): 853-860.

23. Pavlov VA, Wang H, Czura CJ, Friedman SG, Tracey KJ (2003) The cholinergic anti-inflammatory pathway: a missing link in neuroimmunomodulation. Mol Med 9(5-8): 125-134.

24. Goldstein RS, Bruchfeld A, Yang L, Qureshi AR, Gallowitsch-Puerta M, et al. (2007) Cholinergic Anti-
Inflammatory Pathway Activity and High High Mobility Group Box-1 (HMGB1) Serum Levels in Patients with Rheumatoid Arthritis. Mol Med 13(3-4): 210-215.

25. Borovikova LV, Ivanova $S$, Zhang $M$, Yang $H$, Botchkina GI, et al. (2000) Vagus nerve stimulation attenuates the systemic inflammatory response to endotoxin. Nature 405(6785): 458-462.

26. Guarini S, Altavilla D, Cainazzo MM, Giuliani D, Bigiani A, et al. (2003) Efferent Vagal Fibre Stimulation Blunts Nuclear Factor-kappaB Activation and Protects Against Hypovolemic Hemorrhagic Shock. Circulation 107(8): 1189-1194.

27. de Jonge WJ, van der Zanden EP, The FO, Bijlsma MF, van Westerloo DJ, et al. (2005) Stimulation of the vagus nerve attenuates macrophage activation by activating the Jak2-STAT3 signaling pathway. Nat Immunol 6(8): 844-851.

28. Huston JM, Ochani M, Rosas-Ballina M, Liao H, Ochani $\mathrm{K}$, et al. (2006) Splenectomy inactivates the cholinergic antiinflammatory pathway during lethal endotoxemia and polymicrobial sepsis. J Exp Med 203(7): 1623-1628.

29. Wang H, Yu M, Ochani M, Amella CA, Tanovic M, et al. (2003) Nicotinic acetylcholine receptor alpha7 subunit is an essential regulator of inflammation. Nature 421(6921): 384-388.

30. Rosas-Ballina M1, Ochani M, Parrish WR, Ochani K, Harris YT, et al. (2008) Splenic nerve is required for cholinergic antiinflammatory pathway control of TNF in endotoxemia. Proc Natl Acad Sci 105(31): 1100811013.

31. van Maanen MA, Lebre MC, van der Poll T, LaRosa GJ, Elbaum D, et al. (2009) Stimulation of nicotinic acetylcholine receptors attenuates collagen-induced arthritis in mice. Arthritis Rheum 60(1): 114-122.

32. Maini RN, Taylor PC (2000) Anti-cytokine therapy for rheumatoid arthritis. Annu Rev Med 51: 207-229.

33. Tracey D, Klareskog L, Sasso EH, Salfeld JG, Tak PP (2008) Tumor necrosis factor antagonist mechanisms of action: A comprehensive review. Pharmacol Ther 117(2): 244-279.

34. van Maanen MA, Stoof SP, van der Zanden EP, de Jonge WJ, Janssen RA, et al. (2009) The alpha7 nicotinic acetylcholine receptor on fibroblast-like 
synoviocytes and in synovial tissue from rheumatoid arthritis patients: a possible role for a key neurotransmitter in synovial inflammation. Arthritis Rheum 60(5): 1272-1281.

35. Waldburger JM, Boyle DL, Pavlov VA, Tracey KJ, Firestein GS (2008) Acetylcholine regulation of synoviocyte cytokine expression by the $\alpha 7$ nicotinic receptor. Arthritis Rheum 58(11): 3439-3449.

36. Zhou Y, Zuo X, Li Y, Wang Y, Zhao H, et al. (2012) Nicotine inhibits tumor necrosis factor-alpha induced IL-6 and IL-8 secretion in fibroblast-like synoviocytes from patients with rheumatoid arthritis. Rheumatol Int 32(1): 97-104.

37. Bodnar AL, Cortes-Burgos LA, Cook KK, Dinh DM, Groppi VE, et al. (2005) Discovery and Structure-Activity Relationship of Quinuclidine Benzamides as Agonists of $\alpha 7$ Nicotinic Acetylcholine Receptors. J Med Chem 48(4): 905-908.

38. Kitagawa H, Takenouchi T, Azuma R, Wesnes KA, Kramer WG, et al. (2002) Safety, pharmacokinetics, and effects on cognitive function of multiple doses of GTS-21 in healthy, male volunteers. Neuropsychopharmacology 28(3): 542-551.
39. Beckmann J, Schubert J, Morhenn HG, Grau V, Schnettler R, et al. (2015) Expression of choline and acetylcholine transporters in synovial tissue and cartilage of patients with rheumatoid arthritis and osteoarthritis. Cell Tissue Res 359(2): 465-477.

40. Ahmad AM (2007) Recent advances in pharmacokinetic modeling. Biopharm Drug Dispos 28(3): 135-143.

41. Michel V, Yuan Z, Ramsubir S, Bakovic M (2006) Experimental Biology and Medicine Choline Transport for Phospholipid Synthesis. Exp Biol Med 231(1): 490-504.

42. Yabuki M, Inazu M, Yamada T, Tajima $H$, Matsumiya $T$ (2009) Molecular and functional characterization of choline transporter in rat renal tubule epithelial NRK52E cells. Arch Biochem Biophys 485(1): 88-96.

43. Taguchi $C$, Inazu M, Saiki I, Yara $M$, Hara N, et al. (2014) Functional analysis of [methyl-3H] choline uptake in glioblastoma cells: Influence of anti-cancer and central nervous system drugs. Biochem Pharmacol 88(3): 303-312. 